\section{Persistent bradycardia after hypoglycaemia: a case report and a brief literature review}

Beatrice Gasperini, ${ }^{1}$ Pierpaolo Lamanna, ${ }^{1}$

Rocco Serra, ${ }^{1}$ Roberto Montanari, ${ }^{1}$ Antonio Cherubini, ${ }^{2}$ Emma Espinosa ${ }^{1}$

${ }^{1}$ Department of Geriatrics and

Rehabilitation, Santa Croce Hospital, AO Ospedali Riuniti Marche Nord, Fano (PU); ${ }^{2}$ Department of Geriatrics and Italian National Research Centre on Aging (IRCCS-INRCA), Ancona, Italy

\section{Abstract}

Hypoglycaemia can cause cardiac arrhythmias such as QT interval prolongation and ventricular arrhythmias. Supraventricular arrhythmias and sinus bradycardia were rarely reported.

We present the clinical case of an 84year-old man who developed a persistent bradycardia after a hypoglycaemic episode. After restoration of normoglycaemia, bradycardia persisted for almost eighteen hours, without QT prolongation or any symptoms.

Hypoglycaemia is an unusual cause of bradyarrhytmias mainly mediated by neurologic and endocrine systems. Our clinical case supports recent recommendations for more relaxed inpatient glycaemic targets in frail older adults who may be particularly vulnerable to hypoglycaemia and its consequences.

\section{Introduction}

Hypoglycaemia can determine cardiac arrhythmias such as torsade de point, ventricular arrhythmias, and, less frequently, supraventricular arrhythmias. Bradycardia is an unusual cardiac complication. We describe an unusual prolonged bradycardia related to comorbidity and polypharmacy.

\section{Case Report}

EL is an 84-year-old man admitted to hospital for bloody diarrhoea, fever and abdominal pain. His clinical history has been remarkable for rectal ulcerative colitis for three years. He suffered also from hypertensive heart disease with paroxysmal atrial fibrillation (two episodes), vascular cognitive impairment, insulin-treated dia- betes, diverticular disease, prostatic hypertrophy, erythrocytosis in haematological follow-up, impaired mobility, $\mathrm{He}$ is treated with prednisone $5 \mathrm{mg} /$ day, bisoprolol 1.25 $\mathrm{mg}$, mesalazine $800 \mathrm{mg}$, warfarin on INR value, lansoprazole $15 \mathrm{mg}$, insulin glargine 6 units in the morning, rapid-acting insulins 6 units at main meals.

On admission, vital parameters were normal (blood pressure 120/80 mmHg, oxygen saturation $98 \%$ ), temperature was $37.1^{\circ} \mathrm{C}$. The ECG showed sinus rhythm with a heart rate of 78 beats/minute, first degree atrioventricular block (PR 220 msec), right bundle branch block, left anterior fascicular block, QTc interval $420 \mathrm{msec}$ (Bazett's formula) (Figure 1A). His abdomen was lying, painful to deep palpation. Blood tests showed erythrocytosis $\left(8.58 \times 10^{6} \mathrm{mmc}\right.$, hematocrit $\left.61.3 \%\right)$, leukocytosis $(10.60 \times 10$; neutrophils $75 \%)$, moderate renal impairment (eGFR $34 \mathrm{~mL} / \mathrm{min}$ ), increased inflammatory markers (C-protein $>25 \mathrm{mg} / \mathrm{mL}$ ) and mild hypokalemia (3.3 $\mathrm{mEq} / \mathrm{dL}$ ). Apart from the first day, there were no alterations in serum electrolytes, including potassium and calcium, during hospitalization. The abdomen X-Ray and CT scan documented a partial bowel obstruction, with a localized inflammation of the sigma. Ciprofloxacine $200 \mathrm{mg}$ twice a day and metilprednisolone $20 \mathrm{mg}$ twice a day were prescribed and parenteral nutrition was started. Prompt resolution of diarrhoea was obtained, with restoration of regular bowel function, and resolution of pain. However, parenteral nutrition and concomitant use of intravenous steroids required an increase in insulin dose. Along with the remission of abdominal symptoms, oral feeding was restored, parenteral nutrition was suspended and steroid therapy gradually tapered. On the fifth day, the glycemic stick picked at 12 o'clock resulted 121 $\mathrm{mg} / \mathrm{dL}$. Rapid-acting insulin 8 IU was administered, but the patient did not complete the meal. In an hour the patient experienced sudden illness, profuse sweating and a rapid deterioration of the consciousness up to coma. The capillary blood glucose was $40 \mathrm{mg} / \mathrm{dL}$. Blood pressure was $120 / 60 \mathrm{mmHg}$ and heart rate was $36 \mathrm{bpm}$. The ECG showed sinus bradycardia, first degree atrioventricular block (PR unchanged), right bundle branch block, normal QT interval (440 msec corrected) (Figure 1B). Hypoglycaemia was managed with infusion of $50 \mathrm{~mL}$ of $33 \%$ glucose, followed by $500 \mathrm{~mL}$ of $10 \%$ glucose solution with restoration of normal blood glucose levels in five minutes $(100 \mathrm{mg} / \mathrm{dL})$. The consciousness of the patient rapidly improved, with complete resolution of symptoms in about ten minutes but ECG
Correspondence: Beatrice Gasperini, Santa Croce Hospital, AO Ospedali Riuniti Marche Nord, viale Vittorio Veneto 2, 61032 Fano (PU), Italy. Tel.: +39.0721.882309.

E-mail: beatricegasperini@hotmail.com

Key words: Bradycardia; hypoglycaemia; comorbidity.

Acknowledgments: we would like to thank Mrs Elena Pascucci for her kind linguistic revision. Informed consent was obtained from the patient included in the case report.

Conflict of interest: the Authors do not have any conflict of interest.

Received for publication: 31 October 2018. Revision received: 14 January 2019.

Accepted for publication: 15 January 2019.

This work is licensed under a Creative Commons Attribution-NonCommercial 4.0 International License (CC BY-NC 4.0).

(C) Copyright B. Gasperini et al., 2019

Licensee PAGEPress, Italy

Geriatric Care 2019; 5:7670

doi:10.4081/gc.2019.7670

showed a persistent sinus bradycardia to 38 beats/minute with unchanged QT interval. Plasma glycemia remained steadily normal requiring no further correction. A multiparametric monitoring was started. Four hours later the ECG was still unchanged in frequency, PR and QT interval, and heart rate was still 40 beats/minute (Figure 1C). Blood pressure was consistently within normal ranges, while heart rate slowly increased up to 58 beats/minute next morning (18 hours after restoration of normoglycemia). An ECG Holter was started and it showed a stable sinus rhythm with normal heart rate for the whole duration of the recording (minimum night frequency of 60 beats/minute) (Figure 1D).

\section{Discussion}

In older adults with diabetes and established cardiovascular disease the risks associated with hypoglycaemia should be carefully considered. Hypoglycaemia can determine cardiac arrhythmias such as torsade de point, ventricular arrhythmias, and, less frequently, supraventricular arrhythmias. A QT prolongation can occur also during moderate hypoglycemia. ${ }^{1}$

Furthermore, hypoglycaemia is an unusual cause of compromising bradyarrhytmias mainly mediated by neurologic 
and endocrine systems. ${ }^{2}$ The majority of literature in this field consists of case reports. Some cases of bradycardia associated with hypoglycaemia were described in young adults with anorexia or in children who suffered from haemangioma treated with propranolol. Only a few reports concern diabetic older adults.

Pollok described three cases of symptomatic hypoglycaemia complicated with bradycardia. Only one of these regards an older adult; in all cases bradycardia was resolved immediately after the restoring of euglycemic status. ${ }^{2}$

Lindström et al studied six insulindependent subjects without known cardiac diseases. One subject out of six developed bradycardia during severe hypoglycaemia. ${ }^{3}$

Another case report refers the case of a 70 year-old diabetic woman, presenting severe hypoglycaemia, coma, marked sinus bradycardia and QT prolongation. Intravenous administration of glucose and atropine aroused the patient and corrected the bradycardia, but the QT interval was not affected by the treatment. ${ }^{4}$
Chow et al. studied the prevalence of arrhythmias in twenty-five insulin-treated patients (mean age 64 years old) with type2 diabetes and a history of cardiovascular disease. They observed a minimum heart rate during nocturnal hypoglycaemia of 34 beats/minute, with the longest bradycardic period being 156 consecutive beats. When hypoglycaemia was induced with concomitant $\beta$-blockade, a late bradycardia was observed $45 \mathrm{~min}$ after the acute sympathetic reaction at the glucose nadir. ${ }^{5}$

In patients with insulin-dependent diabetes mellitus, a deficiency in glycaemic counterregolation is described, including a modest glucagons secretory response and a reduced response to epinephrine to hypoglycaemia. The duration of diabetes plays a role in decreasing the efficacy of the sympathoadrenal axis $^{6}$ and therefore the occurrence of bradycardia may be linked to an excessive vagal activation after a scarce adrenergic counterregolatory phase.

In previous articles, it was shown that the physiological increase in ventricular frequency in response to insulin-induced hypoglycaemia could be abolished by the use of $\beta$-blockers. Indeed, $\beta$-blockers may completely block the adrenergic reaction to hypoglycaemia and this effect may persist for the duration of the half-life of the $\beta$ blocker.

In our patient, absolute and relative insulin excess was due to the lack of synchronization between meal timing and prandial insulin, polypharmacy, and the interruption of nutritional support. These were the main causes of hypoglycaemia, combined with renal failure, treatment with a quinolone and $\beta$-blocker, which contribute to inhibition of glycogenolysis, gluconeogenesis, and lipolysis. Bradycardia persisted for almost eighteen hours, without QT prolongation or any symptoms and without serum electrolytes modifications. The patient assumed a very low dose of selective $\beta$-blockers, prescribed for paroxysmal atrial fibrillation seven days before the severe hypoglycaemia, but this dose seemed to be sufficient to determine a prolonged bradycardia.

Of note, some authors suggested that
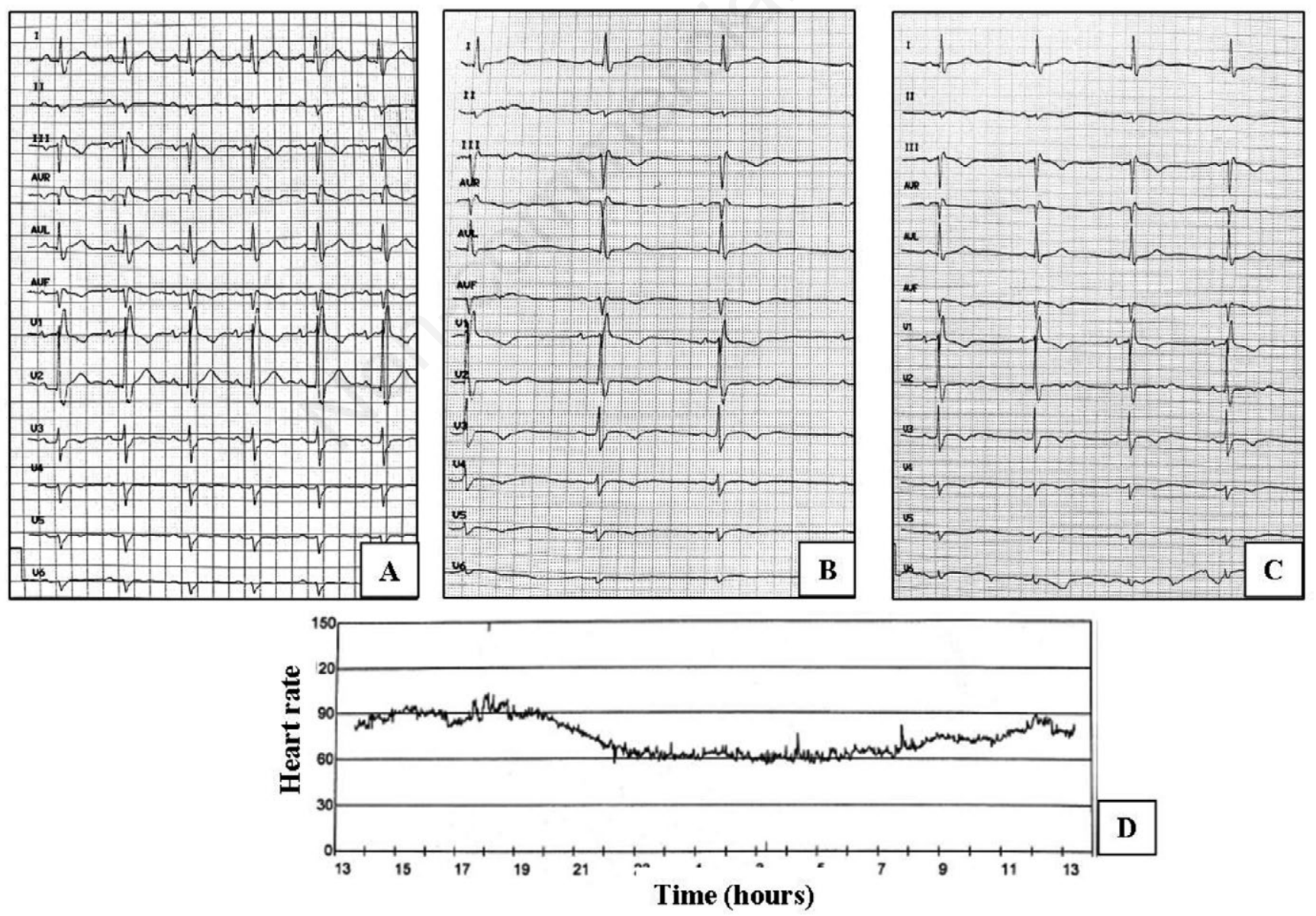

Figure. Patient's ECG: A) At admission; B) during hypoglycaemia; C) four hours after hypoglycaemia; D) ECG Holter report. 
the use of $\beta$-adrenergic blockade markedly reduced cardiac arrhythmias and completely abrogated deaths due to severe hypoglycaemia. ${ }^{7}$ Thus, in our frail patient sinus bradycardia occurred during severe hypoglycaemia and persisted for several hours even without other signs and symptoms, showing atypical manifestation due to polypharmacy and multimorbidity.

\section{Conclusions}

Our clinical case supports recent recommendations on the management of diabetes in older adults. ${ }^{8}$ Indeed, guidelines advocate for more relaxed inpatient glycaemic targets especially in frail older adults who may be particularly vulnerable to side effects such as cardiac complaints.

\section{References}

1. Dominguez LJ, Paolisso G, Barbagallo M. Glucose control in the older patient: from intensive, to effective and safe. Aging Clin Exp Res 2010;22:274-80.

2. Pollock G, Brady WJ Jr, Hargarten S, et al. Hypoglycemia manifested by sinus bradycardia: a report of three cases. Acad Emerg Med 1996;3:700-7.

3. Lindström T, Jorfeldt L, Tegler L, Arnqvist HJ. Hypoglycemia and cardiac arrhythmias in patients with type 2 diabetes mellitus. Diabet Med 1992;9:536-41.

4. Bolognesi R, Tsialtas D, Bolognesi MG, Giumelli C. Marked sinus bradycardia and QT prolongation in a diabetic patient with severe hypoglycemia. J Diabet Complicat 2011;25:349-51.
5. Chow E, Bernjak A, Williams S, et al. Risk of cardiac arrhythmias during hypoglycemia in patients with type 2 diabetes and cardiovascular risk. Diabetes 2014;63;1738-47.

6. Fisher BM, Gillen G, Hepburn DA, et al. Cardiac responses to acute insulininduced hypoglycemia in humans. Am J Physiol 1990;258:H1775-9.

7. Robinson RT, Harris ND, Ireland RH, et al. Comparative effect of human soluble insulin and insulin aspart upon hypoglycemia-induced alterations in cardiac repolarization. $\mathrm{Br} \mathrm{J}$ Clin Pharmacol 2003;55:246-51.

8. Consensus statement by the American Association of Clinical Endocrinologists and American College of Endocrinology on the comprehensive type 2 diabetes management algorithm - 2017 executive summary. Endocr Pract 2017;23:2. 